\title{
A Re-entrant Cavity for Measurement of Complex Permeability in the Very-High-Frequency Region
}

\author{
R. D. Harrington, R. C. Powell, and P. H. Haas
}

\begin{abstract}
A re-entrant cavity, constructed for measuring the initial complex permeability of magnetic toroids in the very-high-frequency region, is described. Many of the electrical and mechanical problems associated with the development of an instrument of this type are considered. The general theory of permeability measurements, using a re-entrant cavity, is discussed, and the associated working equations are derived. Calibration techniques and results of measurements on several samples are also given.
\end{abstract}

\section{Introduction}

The increasing use of magnetic cores for high-frequency inductors and tuning applications has created a demand for information concerning the magnetic properties of materials in the very-high-frequency region. Research and production techniques are thus in need of a rapid and reasonably accurate method for measuring the initial complex permeability of toroidal samples in the region of $100 \mathrm{Mc}$.

Corresponding measurement problems in the frequency region below $30 \mathrm{Mc}$ were solved at the National Bureau of Standards through the development of the radio-frequency permeameter [1]. ${ }^{1}$ Methods used in the analysis of the permeameter are not readily extended into the higher frequency ranges because it is well known that an accurate measurement of radio-frequency permeability in the VHF region requires distributed parameter systems. These techniques usually involve impedance measurements with coaxial lines or cavities containing a sample whose dimensions are small compared to the wave-

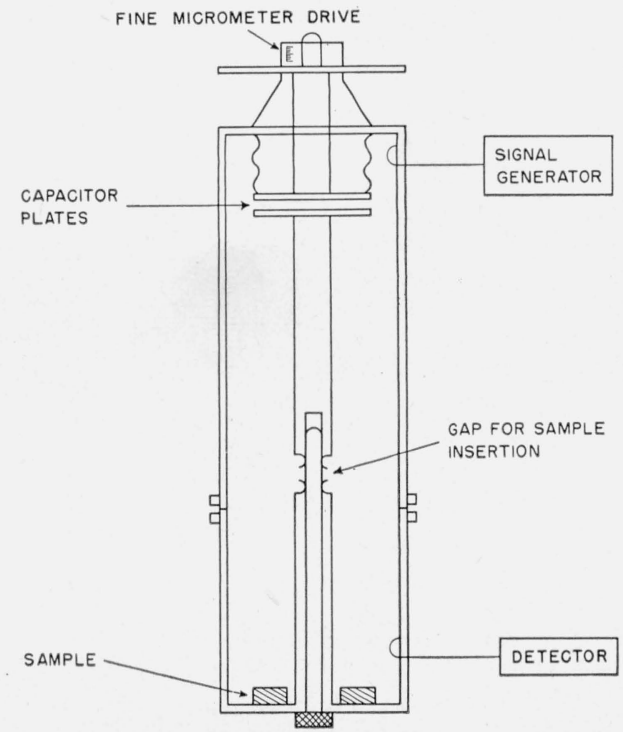

Figure 1. Schematic drawing of re-entrant cavity for determining radio-frequency permeability of magnetic toroids.

\footnotetext{
1 Figures in brackets indicate the literature references at the end of this paper.
}

length within it, and which is placed in a region of high magnetic field. When consideration is given to the study of permeability samples of very low loss, a cavity measurement is most readily utilized because of the attainable high $Q$ of the instrument. For this reason, an investigation was undertaken for the development of a re-entrant resonant cavity for the measurement of initial complex permeability of toroids in the frequency range of 60 to $180 \mathrm{Mc}$. A schematic drawing of a re-entrant cavity of the type developed under this research program is shown in figure 1.

Cavities of this type have been constructed by other investigators $[2,3,4]$, and as such the basic principles of these measurements are fairly well known. The design and analysis of the instrument developed at the National Bureau of Standards differs somewhat from these previous models. A description of the cavity and a brief discussion of the measurement methods and calibration techniques used in the determination of permeability with this instrument are given. Many of the electrical and mechanical problems that arise in the construction of a re-entrant cavity for magnetic measurements are discussed.

\section{Description of Instrument}

A re-entrant cavity is, essentially, a shorted section of coaxial transmission line with a lumped capacitance at one end, as can be seen in figure 1 . The capacitor for the Bureau instrument consists of two parallel capacitor plates whose capacitance or separation distance can be adjusted by means of the vernier micrometer drive shown near the top of figures 1 and 2 . The complex permeability of a sample is determined from a measurement of the variation in $Q$ and the change in the resonant capacity of the cavity when a small toroidal sample is placed at the bottom of the instrument in a region of high magnetic field. It should be noted that measurements can also be obtained by holding the capacity fixed and determining the change in resonant frequency upon insertion of a sample [2]. However, this variation in frequency is quite small, and its measurement requires elaborate frequencymeasuring equipment, such as counters or heterodyne systems. As an instrument was desired that 
would give a fairly accurate permeability measurement for laboratory as well as production and quality control purposes, emphasis was placed on capacity-variation techniques that require only an oscillator and detector as associated equipment.

If the cavity is to cover a reasonably large frequency range, the tuning capacitance must be capable of a variation of the order of 10 or 20 to 1 . However, to increase the frequency coverage further, the instrument was constructed in two lengths, as shown in figure 2. The longer instrument is used in the 60 - to $110-\mathrm{Mc}$ region, whereas the short cavity resonates in the 110 - to $180-\mathrm{Mc}$ region. The flange in the middle of the cavity facilitates the removal and interchange of the two bottom sections of different lengths. This makes it necessary to construct only one top unit, consisting of the capacitor plates and vernier unit.

In order to avoid making the unit excessively long, the maximum capacitance must be large if low frequencies are to be obtained. This capacitance was obtained on the National Bureau of Standards instrument by using capacitor plates 1.5 in. in diam.eter. Placing a toroid at the bottom of the cavity would require slipping it over these plates. This is undesirable as few representative cores have inside dimensions of $1.5 \mathrm{in}$. To insert the core through

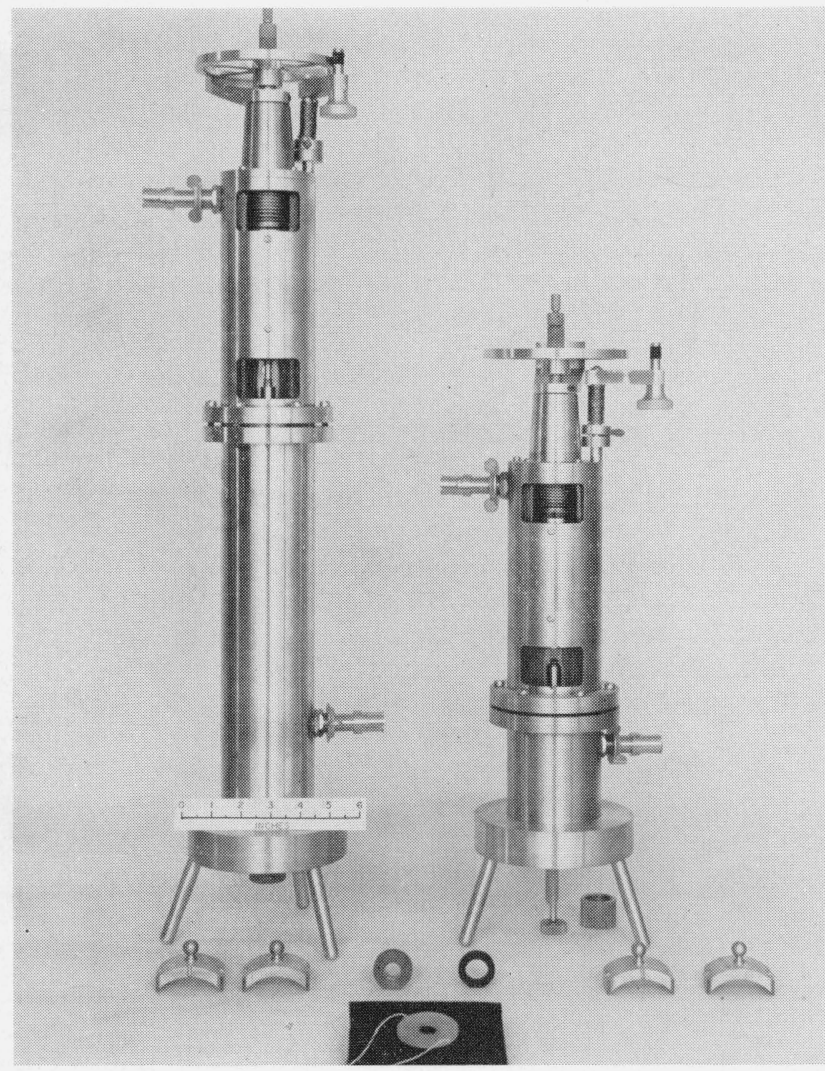

FIGURE 2. Re-entrant cavities for very-high-frequency permeability measurements and typical samples measured with these instruments. the bottom of the cavity would require removing some type of shorting plug in the bottom section of the instrument, which, in view of the need of a high reproducible $Q$, is impractical. That region of the cavity is, of course, a current maximum, and changes of resistance at the bottom cause large changes in cavity losses.

This problem was solved by using a hollow center conductor through which a copper rod could slide. A gap in the hollow conductor was placed at a considerable distance above the bottom of the cavity, and the center copper rod completed the circuit by making contact with beryllium-copper fingers on the inside of the hollow conductor. This opening in the center conductor may be seen in the lower window of the two cavities in figure 2 and, in more detail, in figure 3. A core may be inserted by removing the cap at the bottom of the cavity and withdrawing the copper rod. The sample is then placed on a small plastic platform held by nylon threads and

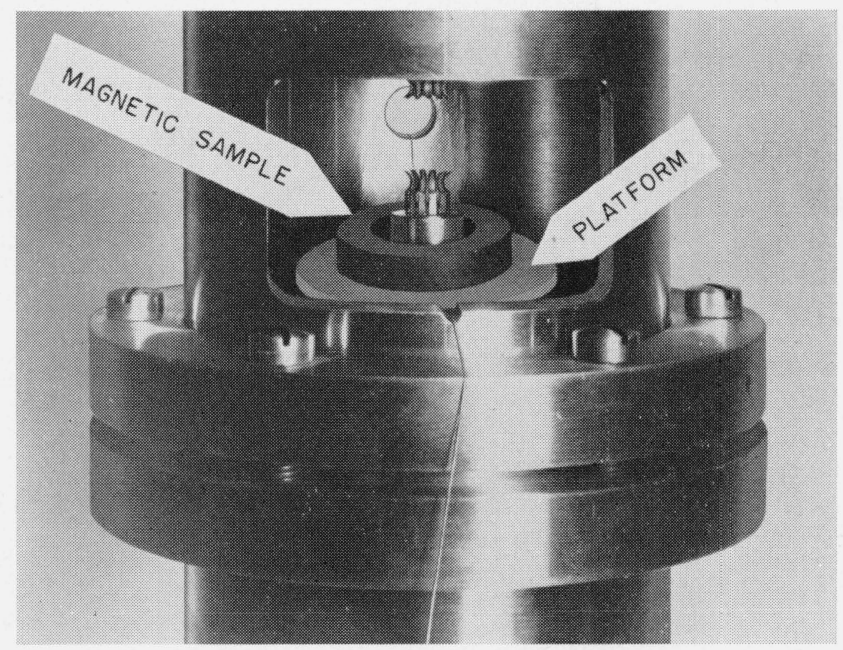

FiguRe 3. Lower window of re-entrant cavity showing technique for inserting sampl?.

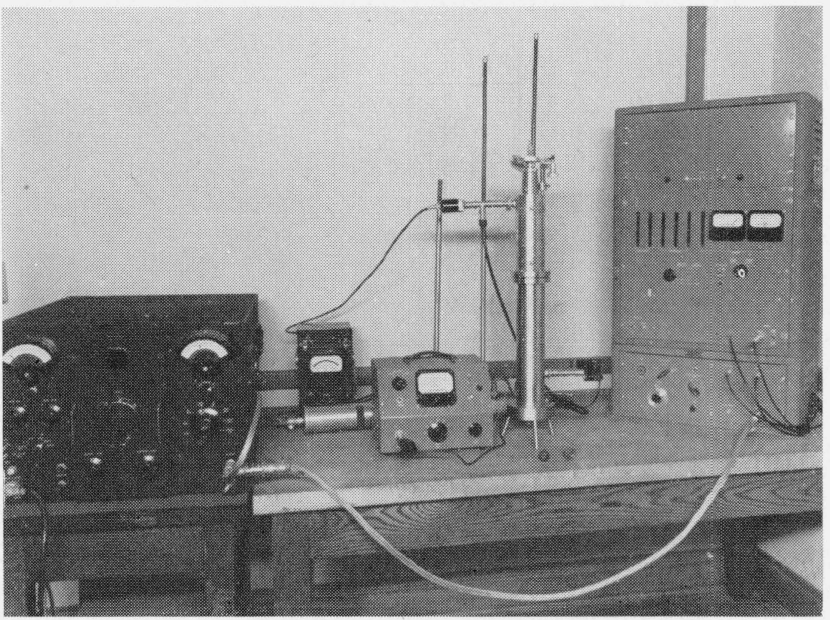

FIGURE 4. Re-entrant cavity and associated equipment. 
lowered to the bottom of the cavity. This platform and a typical sample can be seen in figure 3 . This method of sample insertion thus allows the center conductor to have a much smaller diameter than the condenser plates, and, of course, a more reproducible $Q$ is obtained.

The capacitor plates can be seen in the top window of the cavities in figure 2 . The removable window readily facilitates the cleaning and inspection of the condenser as the capacitance is quite sensitive to dust particles that may collect on the surfaces of the plates. The lower condenser plate is held in place by two dielectric slabs, and a bellows connection is made with the top plate in order to avoid any sliding contacts. The capacitor plates are lapped to within $0.0001 \mathrm{in}$. total indicator reading. The micrometer wheel can be read to 0.0001 in. directly and a vernier to $0.00001 \mathrm{in}$. This accurate dimensioning is necessary as small capacitance differences are used in the calculations. The calibration of the capacitor will be explained later.

Coupling to the cavity is obtained through two adjustable current loops. The input loop is located near the top of the cavity, and the pickup loop for the detector is placed a short distance above the sample, at the bottom of the instrument. Coupling must be loose in order that the input voltage and frequency remain unaffected as the cavity is tuned throughout the resonance curve. A $1 / 32$-amp fuse is used as a bolometer element in order to obtain a better square law characteristic than is possible with a crystal.

A typical equipment set up is shown in figure 4 . A signal modulated with a 1,000-cps signal is passed through a filter and three-stub tuner. The input voltage is monitored with a vacuum tube voltmeter in order to assure loose coupling. The output is fed directly to a bolometer and amplifier. The frequency counter shown in the figure is used for calibration of the $Q$ of the cavity. Once the $Q$ calibration has been completed, the counter is no longer essential for a measurement and is removed from the circuit. The calibration details are given later.

\section{Analysis}

It has already been stated that a permeability measurement is based on the change in the inductive properties of the cavity when a sample is placed in a region of high magnetic field. The method is essentially a perturbation technique that enables one to disregard the effect of the dielectric constant of the specimen as long as the thickness of the sample is small compared to the wavelength within it. It should be noted that this situation is satisfied in the VHF region by many of the samples manufactured commercially. In all cases, however, one must still give consideration to the problems that may arise with certain samples because of dimensional resonance [5].

Consider the inner and outer radii of the internal volume of the coaxial section of the re-entrant cavity to be $\mathrm{A}$ and $\mathrm{B}$, respectively. Let $a, b$, and $h$, respectively, be the corresponding inner radius, outer radius, and height of the sample being considered. If the thickness of the sample is small compared to the wavelength within it, and if the sample is placed against the shorted end of the cavity in a region of high magnetic field, one may consider the electric energy stored in the sample as negligible compared to the stored magnetic energy, and write for the latter quantity

$$
U_{H 1}=\frac{1}{2}\left(\mu^{\prime}-1\right) L_{a}\left|I_{1}\right|^{2}
$$

where $L_{a}$ is the geometrical or equivalent air inductance of a sample of permeability $\mu^{\prime}$ and is given by $L_{a}=0.4606 h \log _{10}(b / a) \times 10^{-6}$ henries, where $h$ is in meters. $I_{1}$ is the current flowing in the vicinity of the sample and is essentially constant over the distance $h$.

If we similarly consider the annular region of the empty cavity of radii $A$ and $B$ and height $\Delta l$ located at the shorted end of the instrument, where $\Delta l$ is sufficiently small, so that the electric energy in this region is also negligibly small, then one may write for the stored magnetic energy for this case

$$
U_{H 2}=\frac{1}{2} \frac{\mu_{0}}{2 \pi} \ln \frac{B}{A}\left|I_{1}\right|^{2} \Delta l,
$$

as the inductance per unit length of a uniform coaxial transmission line is given by

$$
L=\frac{\mu_{0}}{2 \pi} \ln \frac{B}{A},
$$

where $\mu_{0}$ is the permeability of free space.

It can be seen that there will be some value of $\Delta l$ for which. $U_{H 1}=U_{H 2}$. By equating eq (1) and (2), this value of $\Delta l$ is found to be

$$
\Delta l=\left(\mu^{\prime}-1\right) \frac{2 \pi L_{a}}{\mu_{0} \ln \frac{B}{A}}
$$

This equation essentially relates the effect of inserting a sample of permeability $\mu^{\prime}$ into a region of high magnetic field in the cavity to a virtual change in length, $\Delta l$, of the instrument where both the sample thickness and $\Delta l$ are small compared to the wavelength being considered.

This change may be transformed into a corresponding change in the lumped capacitance, $C$, located a distance, $l$, from the short by means of the approximate relation describing a short-circuited high- $Q$ transmission line with a lumped capacitance at one end, which is given by

$$
Z_{0} \tan \beta l=\frac{1}{\omega C},
$$

where

$Z_{0}=$ characteristic impedance of the cavity considered as a coaxial transmission line

$$
\begin{aligned}
& \beta=2 \pi / \text { wavelength } \\
& \omega=\text { angular frequency. }
\end{aligned}
$$


Holding the frequency constant and differentiating, we obtain

$$
d l=-\frac{\omega Z_{0}}{\beta} \sin ^{2} \beta l d C .
$$

Substituting the value of $\Delta l$ found in eq (3) for $d l$ in the above equation, we get

$$
\left(\mu^{\prime}-1\right) \frac{2 \pi L_{a}}{\mu_{0} \ln \frac{B}{A}}=-\frac{\omega Z_{0}}{\beta} \sin ^{2} \beta l d C .
$$

Solving for $\mu^{\prime}-1$ and using the expression for the characteristic impedance of a coaxial transmission line $Z_{0}=(1 / 2 \pi) \sqrt{\mu_{0} / \epsilon_{0}} \ln (B / A)$, where $\epsilon_{0}$ is the permittivity of free space, we obtain

where

$$
\mu^{\prime}-1=-\frac{d C}{k_{2} L_{a}},
$$

$$
k_{2}=\left(Z_{0}^{2} \sin ^{2} \beta l\right)^{-1} .
$$

The above relation gives the real permeability in terms of $d C$, which is the change in capacity necessary to reresonate the cavity upon insertion of the sample. The evaluation of $k_{2}$ is explained later.

The working equation for the measurement of the imaginary part of the permeability $\left(\mu^{\prime \prime}\right)$ may be obtained directly from an analysis of the energy associated with the magnetic field in the cavity. If the usual relations for the magnetic field energy stored, as well as that dissipated per cycle in both the cavity and the sample are substituted in the corresponding expression for the $Q$ of the loaded cavity, a relation is obtained that readily leads to the expression ${ }^{2}$

$$
\mu^{\prime \prime}=\frac{1}{Q_{c}}\left[\left(\mu^{\prime}-1\right) \frac{Q_{c}}{Q_{F}}+\frac{k_{1}}{L_{a}}\left(\frac{Q_{c}}{Q_{F}}-1\right)\right]
$$

where

$$
k_{1}=\frac{Z_{0}\left[\frac{1}{2} \sin 2 \beta l+\beta l\right]}{2 \omega},
$$

and

$$
\begin{aligned}
& Q_{c}=\text { quality factor of empty cavity } \\
& Q_{F}=\text { quality factor of cavity plus sample. }
\end{aligned}
$$

For a high-Q loaded cavity, the ratio of Q's may be replaced by the corresponding voltage ratio $V r=V_{c} / V_{F}$, which is the ratio of the voltage output of the empty cavity to the output of the loaded cavity. Thus, the equation for $\mu^{\prime \prime}$ is given by

$$
\mu^{\prime \prime}=\frac{1}{Q_{c}}\left[\left(\mu^{\prime}-1\right) V r+\frac{k_{1}}{L_{a}}(V r-1)\right] .
$$

We therefore see that once $\mu^{\prime}-1$ has been determined for a specimen, the only additional information necessary for calculating the magnetic loss is the voltage ratio $V r$, the $Q$ of the unloaded cavity,

\footnotetext{
2 See appendix.
}

and the value of the quantity $k_{1}$, which is discussed shortly.

\section{Calibration of Cavity}

The re-entrant cavity is calibrated directly against the primary standard of Radio-Frequency Permeability at the National Bureau of Standards, which is a precision variable-length coaxial line. There are essentially four quantities on the re-entrant cavity that require calibration.

1. The vernier micrometer drive, which must be calibrated in terms of capacitance.

2. The quantity $k_{2}$ in eq (5), which determines the real part of the permeability.

3. The value of $k_{1}$ in eq (8), which is the working equation for the magnetic loss measurement.

4. The $Q$ of the unloaded cavity.

It is not necessary to have a calibration curve for $Q$ if a frequency counter or similar instrument is readily available. However, as previously mentioned, it is preferred that measurements be obtained without relying on frequency-measuring instruments.

Consider first the capacitance calibration. The vernier micrometer could be evaluated in terms of the calculated capacitance between the condenser plates. However, the effects of fringing capacitance must not be neglected. The capacitor is thus calibrated at $1,000 \mathrm{cps}$, using extremely sensitive capacitance standards. This is done by removing the lower door in the cavity and opening the center conductor circuit by withdrawing the removable copper rod. The lower capacitor plate is now isolated from the body of the cavity and held in place by dielectric disks. The lower end of that portion of the center conductor in contact with the bottom capacitor plate may be seen in the lower window of the cavity in figures 2 and 3 . An external wire connection may be made with the lower capacitor plate at this point. The upper capacitor plate is in contact with the body of the cavity. It is thus possible to connect the variable capacitor on the cavity in parallel with a precision variable capacitance standard. This parallel arrangement is placed across one arm of a capacitance bridge, and increments of capacitance in the cavity capacitor are compared directly with corresponding increments in the standard capacitor. The calculated capacitance of the variable capacitor in the cavity for a plate separation larger than that usually encountered in measurements is taken as a reference level. The exact location of this reference point is somewhat arbitrary as one is concerned only with differences in capacitance in this instrument. The above data enables a curve of capacitance versus vernier distance to be obtained; however, the capacitance covers a rather large range, which makes it difficult to obtain a precise graph. This difficulty is avoided by plotting only corrections to the calculated capacitance, which is obtained from the formula

$$
C=\frac{\epsilon_{0} A}{d}
$$


where $\epsilon_{0}$ is the permittivity of free space, and $A$ and $d$ are the cross-sectional area and separation distance, respectively, of the capacitor plates.

The expressions for the quantities $k_{1}$ and $k_{2}$ have already been given, but are repeated below:

$$
\begin{gathered}
k_{2}=\left(Z_{0}^{2} \sin ^{2} \beta l\right)^{-1} \\
k_{1}=\frac{Z_{0}\left[\frac{1}{2} \sin 2 \beta l+\beta l\right]}{2 \omega} .
\end{gathered}
$$

In order to evaluate these quantities, $Z_{0}$ is first calculated from the usual expression for the characteristic impedance of a coaxial transmission line $\left(Z_{0}=138 \log _{10}(B / A)\right.$, where $B$ and $A$ are the outer and inner radii of the cavity). The value of the capacitance between the electrodes is then obtained at a low frequency, where the electrode separation is small and the resulting capacitance large enough to account for most of the cavity capacitance. Equation (4) is then used with the above values of $Z_{0}$ and $C$ to calculate a value of $l$, the effective length of the cavity. A theoretical curve of $k_{2}$ and $k_{1}$ versus frequency may now be obtained. The operating eq (5) and (8) are solved for $k_{1}$ and $k_{2}$ in terms of $\mu^{\prime}, \mu^{\prime \prime}, d C, Q_{c}$, and $V r$. The $\mu^{\prime}$ and $\mu^{\prime \prime}$ are obtained from a measurement of a sample in the primary standard. The remaining quantities $d C$, $Q_{c}$, and $V r$ are obtained from a measurement of the same sample, using the re-entrant cavity. This data is obtained at one frequency for each of the two different length cavities. The resulting experimental values of $k_{1}$ and $k_{2}$ are then used to adjust the theoretical values. It can be seen that this procedure gives an effective value for the characteristic impedance of the cavity.

The $Q$ of a resonant circuit may be obtained from either of the relations

$$
Q=\frac{f_{0}}{\Delta f}=\frac{2 C_{0}}{\Delta C}
$$

where $f_{0}$ and $C_{0}$ are the resonant frequency and total effective capacity, and $\Delta f$ and $\Delta C$ are corresponding $3-\mathrm{db}$ band widths. In the case of a distributed parameter system such as a cavity, the total effective capacitance cannot be calculated. Thus, the frequency relation is more meaningful.

However, the $Q$ of a cavity is quite sensitive to temperature variations, and a more accurate answer is obtained if the $Q$ could be obtained at the time of each measurement instead of using one original calibration of $Q$ versus frequency. In order that only capacitance variations be necessary for a measurement, it was decided to make an initial measurement of $\Delta f, f_{0}$, and $\Delta C$. Equation (11) can then be used to calculate $2 C_{0}$, which may be plotted as a calibration curve. Any future determination of $Q$ may then be obtained from the calibration curve and a measurement of $\Delta C$.

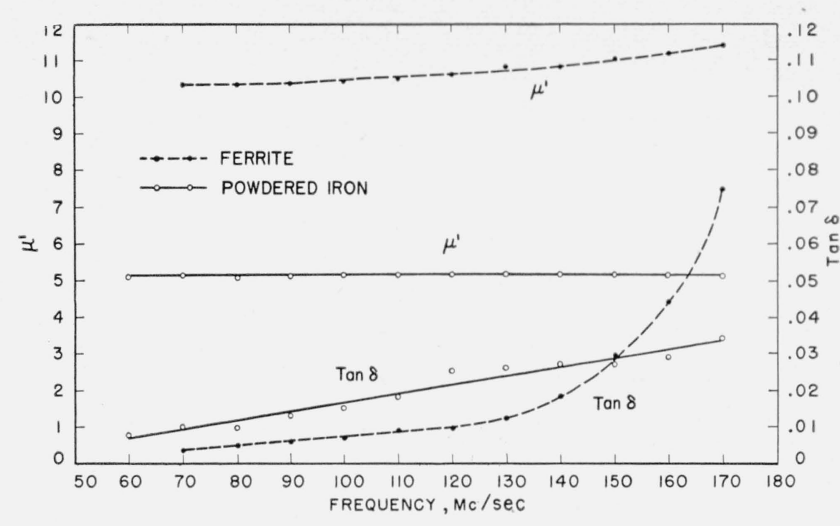

Figure 5. Typical data obtained with re-entrant cavity, using two different samples.

\section{Measurements and Results}

The cavity was normalized against the primary standard by using a sample having a permeability in the order of 8 , which is a typical value for several samples in this frequency range. A comparison of results obtained at $130 \mathrm{Mc}$, using three samples measured in the primary standard and re-entrant cavity, are shown in table 1.

\begin{tabular}{|c|c|c|c|c|}
\hline \multirow{2}{*}{ Sample } & \multicolumn{2}{|c|}{ Primary standard } & \multicolumn{2}{|c|}{ Re-entrant cavity } \\
\hline & $\mu^{\prime}$ & $\tan \delta=\frac{\mu^{\prime \prime}}{\mu^{\prime}}$ & $\mu^{\prime}$ & $\tan \delta$ \\
\hline 1 & $\begin{array}{c}8.102 \\
10.645 \\
5.08\end{array}$ & $\begin{array}{r}0.067 \\
.090 \\
.027\end{array}$ & $\begin{array}{l}8.119 \\
10.768 \\
5.16\end{array}$ & $\begin{array}{r}0.065 \\
.083 \\
.034\end{array}$ \\
\hline
\end{tabular}

TABLE 1. Comparison of results, using a re-entrant cavity and primary standard at $130 \mathrm{Mc}$

A typical curve of permeability versus frequency, using the re-entrant cavity, is shown in figure 5 for a powdered-iron sample and a ferrite.

The above data and curves show the instrument to be capable of good precision and fairly accurate results. This conclusion, along with the simple measurement procedure, gives strong support to the use of a re-entrant cavity for very-high-frequency permeability measurements.

\section{References}

[1] P. H. Haas, A radio frequency permeameter, J. Research NBS 51, 221 (1953) RP2454.

[2] H. J. Lindenhovius and J. C. van der Breggen, The measurement of permeability and magnetic losses of non-conducting ferromagnetic material at high frequencies, Philips Research Reports 3, 37-45 (Feb. 1948).

[3] D. Polder, Ferrite materials, Proc. Inst. Elec. Engrs. (London), 97II, 246-256 (1950).

[4] C. M. van der Burgt, M. Gevers, and H. P. J. Wijn, Measuring methods for some properties of ferroxcube materials, Philips Tech. Rev. 14, 245-280 (March 1953).

[5] Brochman, Dowling, and Steneck, Dimensional effects resulting from a high dielectric constant found in a ferromagnetic ferrite, Phys. Rev. $7 \boldsymbol{y}, 85-93$ (Jan. 1950). 


\section{Appendix. Derivation of Loss Equation}

Definition of terms:

$V=$ volume of cavity including space occupied by sample.

$v=$ volume of sample.

$E c_{1}=$ total energy stored in volume $V-v$.

$E s_{1}=$ total energy stored in space occupied by sample of volume $v$.

$E c_{2}=$ energy dissipated per cycle in cavity without sample.

$E s_{2}=$ energy dissipated per cycle in space occupied by sample of volume $v$.

$A, B, l=$ inner radius, outer radius, and length of cavity.

$a, b, h=$ inner radius, outer radius, and height of sample.

$t=$ length measured along axis of cavity or sample.

$r=$ radial distance measured in cavity or sample.

$\beta=$ phase constant in cavity.

$Q_{c}=Q$ of empty cavity.

$Q_{s}=Q$ of sample.

$Q_{F}=Q$ of cavity plus sample.

The $Q$ of the loaded cavity may be written

$$
Q_{F}=2 \pi \frac{E c_{1}+E s_{1}}{E c_{2}+E s_{2}}
$$

Let

$$
H=\frac{H_{0}}{r} \cos \beta t
$$

where $H$ is the magnetic field at a distance $t$ from the shorted end of the cavity.

If we assume the thickness of a sample is small compared to the wavelength within it, then we may consider $\cos \beta t$ as being essentially unity over the dimensions of the sample, as the sample is always located at the shorted end of the instrument.

Thus

$$
\begin{gathered}
E c_{1}=\frac{\mu_{0}}{2} \int_{V} \frac{H_{0}^{2}}{r^{2}} \cos ^{2} \beta t d V-\frac{\mu_{0}}{2} \int_{v} \frac{H_{0}^{2}}{r^{2}} d v \\
=\pi \mu_{0} H_{0}^{2}\left[\frac{1}{\beta}\left(\ln \frac{B}{A}\right)\left(\frac{1}{2} \beta l+\frac{1}{4} \sin 2 \beta l\right)-h \ln \frac{b}{a}\right] \\
E s_{1}=\frac{\mu_{0}}{2} \int_{v} \frac{\mu^{\prime} H_{0}^{2}}{r^{2}} d v=\pi \mu_{0} H_{0}^{2} \mu^{\prime} h \ln \frac{b}{a} \\
E c_{2}=\frac{2 \pi}{Q_{c}}\left(\frac{\mu_{0}}{2} \int_{V} \frac{H_{0}^{2}}{r^{2}} \cos ^{2} \beta t d V\right),
\end{gathered}
$$

and

$$
E s_{2}=\frac{2 \pi E s_{1}}{Q_{s}}
$$

Let

$$
K=\left(\frac{1}{2} \beta l+\frac{1}{4} \sin 2 \beta l\right)
$$

Substituting the above values in eq (12), we obtain

$$
Q_{F}=\frac{\frac{1}{\beta}\left(\ln \frac{B}{A}\right) K-\ln \frac{b}{a} h+\mu^{\prime}\left(\ln \frac{b}{a}\right) h}{\frac{1}{Q_{c} \beta} \ln \frac{B}{A} K+\mu^{\prime} \frac{1}{Q_{s}} \ln \frac{b}{a} h} .
$$

Solving for $\mu^{\prime \prime}=\mu^{\prime} / Q_{s}$, we get

$$
\begin{gathered}
\mu^{\prime \prime}=\frac{1}{\beta h} \frac{\ln \frac{B}{A}}{\ln \frac{b}{a}} \frac{K}{Q_{F}}+\left(\mu^{\prime}-1\right) \frac{1}{Q_{F}}-\frac{1}{Q_{c} \beta h} \frac{\ln \frac{B}{A}}{\ln \frac{b}{a}} K \\
\mu^{\prime \prime}=\frac{1}{Q_{c}}\left[\left(\mu^{\prime}-1\right) \frac{Q_{c}}{Q_{F}}+\frac{1}{\beta h} \frac{\ln \frac{B}{A}}{\ln \frac{b}{a}} K\left(\frac{Q_{c}}{Q_{F}}-1\right)\right] .
\end{gathered}
$$

Now

$$
\beta=\omega \sqrt{\mu_{0} \epsilon_{0}} .
$$

The geometrical inductance of the sample is

$$
L_{a}=\frac{\mu_{0}}{2 \pi}\left[\ln \frac{b}{a}\right] h .
$$

The characteristic impedance of the cavity is

$$
Z_{0}=\frac{1}{2 \pi} \sqrt{\frac{\mu_{0}}{\epsilon_{0}}} \ln \frac{B}{A} .
$$

Thus

$$
\frac{1}{\beta h} \frac{\ln \frac{B}{A}}{\ln \frac{b}{a}}=\frac{1}{\omega \sqrt{\mu_{0} \epsilon_{0}}} \frac{2 \pi Z_{0} \sqrt{\frac{\epsilon_{0}}{\mu_{0}}}}{2 \pi \frac{L_{a}}{\mu_{0}}}=\frac{Z_{0}}{\omega L_{a}} .
$$

Substituting in eq (17)

$$
\mu^{\prime \prime}=\frac{1}{Q_{c}}\left[\left(\mu^{\prime}-1\right) \frac{Q_{c}}{Q_{F}}+\frac{Z_{0} K}{\omega L_{a}}\left(\frac{Q_{c}}{Q_{F}}-1\right)\right] .
$$

Letting

$$
k_{1}=\frac{Z_{0} K}{\omega}=\frac{Z_{0}}{2 \omega}\left(\frac{1}{2} \sin 2 \beta l+\beta l\right),
$$

we obtain eq (6)

$$
\mu^{\prime \prime}=\frac{1}{Q_{c}}\left[\left(\mu^{\prime}-1\right) \frac{Q_{c}}{Q_{F}}+\frac{k_{1}}{L_{a}}\left(\frac{Q_{c}}{Q_{F}}-1\right)\right] .
$$

Boulder, October 11, 1955. 